\title{
Duoc UC: \\ UNA EXPERIENCIA EN LA \\ FORMACIÓN TÉCNICA Y PROFESIONAL
}

Marcelo von Chrismar*

Francisco Valdés** 


\section{RESUMEN}

La baja productividad de la mano de obra y la falta de incorporación oportuna de las tecnologías a todas las actividades del país, constituyen, sin duda, un freno para nuestro desarrollo. Las instituciones de educación superior tienen la responsabilidad de aportar en ambos aspectos, a través de la preparación de los cuadros técnicos y profesionales que serán los encargados de dirigir los procesos productivos de bienes y servicios.

El modelo educativo de DuocUC tiene como objetivo preparar a los estudiantes para el mundo del trabajo, a través de programas diseñados con una orientación específica a fin de alcanzar las competencias laborales. Para ello cuenta con la asesoría de Consejos empresariales, constituidos por personas directamente relacionadas con las actividades en las que se desempeñarán los futuros egresados. La entrega de conocimientos teóricos, junto con la aplicación práctica de los mismos, en laboratorios que permiten simular la realidad que encontrarán en su desempeño profesional, les ofrece asimismo garantías de experiencia en el uso de las tecnologías actuales.

\section{ABSTRACT}

One of the obstacles to development faced by Chile is, undoubtedly, the low productivity of labor and the lack of a timely inclusion of new technologies to all the activities conducted in the country. Higher education centers have a duty to make a contribution in both respects, by preparing technical and professional cadres, who will be responsible for directing the process of producing goods and services in the country.

The educational model implemented by DuocUC is aimed at training students for the world of work through programs designed especially to achieve labor competency. For this purpose, it relies on the advice of the Consejos empresariales (Business Councils), formed by people directly related to activities in which future graduates will work. The theoretical knowledge that these students acquire, together with a practical application of what they have learned in laboratories that allow simulating the reality they will find in the world of work, provides a guarantee of experience in the use of current technologies. 


\section{Duoc UC: \\ UNA EXPERIENCIA EN LA FORMACIÓN TÉCNICA Y PROFESIONAL}

Tradicionalmente, la formulación de programas orientados a la formación para el trabajo se abordaba descomponiendo las tareas de una ocupación determinada en operaciones simples. Posteriormente -a través de un proceso semejante al de la ingeniería reversa, utilizada en diseño- se organizaba el curso, enseñando las operaciones en orden creciente de complejidad. Con el correr de los años esta metodología fue dificultando cada vez más el diseño de los cursos, ello debido a la necesidad de incorporar constantemente nuevas tecnologías, con miras a entregar la educación que requería la industria.

Adicionalmente, la flexibilización en el trabajo (que implica el desempeño alternativo de varias ocupaciones calificadas), el cambio y rotación del lugar de trabajo, el avance tecnológico creciente, el desarrollo organizacional de las tareas productivas, el aumento de la complejidad e interdependencia de las labores que lo constituyen, ha obligado a la participación de diferentes especialidades en una misma actividad, lo cual ha llevado a la necesidad de efectuar trabajos en equipos de personas. Ello ha significado que la metodología de diseño de programas educacionales evolucione, a partir de las calificaciones hacia unos currículos orientados a entregar "competencias laborales", definidas como la combinación integrada de conocimientos, habilidades y actitudes que conduzcan a un desempeño adecuado y oportuno en una ocupación dada.

Asimismo, de un tiempo a esta parte, las empresas han variado su perspectiva al seleccionar a sus trabajadores. En efecto, ya no lo hacen considerando las necesidades específicas de un determinado puesto de trabajo; más bien se preocupan de los requerimientos para desempeñar una serie de ocupaciones que exigen competencias semejantes a quienes las desempeñan. 
Por mucho tiempo se pensó que para asegurar el éxito económico en la vida bastaba con poseer un "cartón universitario". Hoy, sin embargo, lo que realmente vale para asegurar la empleabilidad es contar con competencias laborales valoradas como tales por los empleadores y que al mismo tiempo sean demostrables. En este aspecto radica la ventaja de las carreras cuyo objetivo es preparar a los estudiantes para el mundo del trabajo, puesto que sus programas han sido diseñados con una orientación específica para alcanzar las competencias que la industria requiere de sus profesionales y técnicos. No hay que olvidar que el desarrollo de los países demanda en forma creciente el concurso de profesionales y técnicos especializados, capaces de responder con éxito a las demandas concretas de la industria.

Los requerimientos necesarios para desarrollarse con éxito en el mundo del trabajo, exigen de las instituciones de educación superior el logro de un "saber hacer" laboral, con dos componentes básicos: competencias o capacidades de acción y fundamentos o habilidades que permiten alcanzarlas.

Las primeras son de cinco tipos:

a) Competencias para el uso de recursos: identificar, organizar, planear y asignar recursos;

b) Competencias interpersonales: trabajar con otros;

c) Competencias para el uso de información: adquirir y utilizar la información;

d) Competencias para el manejo de sistemas: entender interrelaciones complejas;

e) Competencias para el uso de tecnologías: trabajar con una variedad de tecnologías.

Para poder dominar estas competencias es necesario contar con fundamentos que se expresan en habilidades y cualidades de tres tipos:

a) Habilidades básicas: leer, escribir, escuchar, comunicar, realizar cómputos, etcétera;

b) Habilidades del pensamiento: tomar decisiones, resolver problemas, trabajar con lógica simbólica, procesar símbolos, aplicar nuevos conocimientos extrapolando situaciones o sacando conclusiones, etcétera;

c) Cualidades personales: responsabilidad, autoestima, sociabilidad, autocontrol, integridad, honestidad. 
Así entonces, un factor clave para el diseño de planes de estudio y carreras en el modelo educativo de un instituto profesional como el DuocUC, es la capacidad para detectar las necesidades reales que, dada la evolución actual de las empresas, se requieren para el desarrollo de los diferentes tipos de labores tanto en lo que se refiere a las especialidades requeridas, como al perfil de competencias laborales. Para seleccionarlas se deben considerar las capacidades y habilidades antes enunciadas, en función de los requerimientos propios del mundo del trabajo y establecer con base en ellas las competencias que permitan la programación educacional desde la demanda y no desde la oferta. De la misma forma se debe proceder con las tecnologías, que representan un factor clave para el diseño de los planes de estudios y carreras.

Para ello, y con el fin de enriquecer la experiencia que se puede recoger directamente de los propios profesores, en las diferentes escuelas del DuocUC se han conformado Consejos empresariales, compuestos por destacados profesionales y empresarios en cada una de las especialidades, quienes aportan su visión, conocimientos y experiencia.

El éxito en la inserción de los egresados en sus puestos de trabajo queda garantizado, tanto porque en la selección de las carreras y sus especialidades está considerada la demanda real por técnicos y profesionales, como, porque los correspondientes currículos se diseñan con una orientación hacia las competencias laborales.

Por otra parte, la metodología de enseñanza-aprendizaje que se utiliza en DuocUC, se basa en la ejercitación práctica en laboratorios, en los cuales se simula la realidad que los alumnos encontrarán en las empresas durante su desempeño profesional, al mismo tiempo que se van entregando los fundamentos teóricos de las diferentes materias: es decir, se basa en el "aprender-haciendo". Esta metodología facilita también la incorporación de los egresados a su trabajo, ya que los problemas y soluciones que allí enfrenten les resultarán familiares.

Otro aspecto fundamental a considerar en la formación para el trabajo es la relación entre la competitividad y el tipo de educación. Las componentes de la tasa de crecimiento del PIB de un país son el capital, la Productividad Total de los Factores (PTF) y el trabajo. En el caso de Chile, el cuadro siguiente muestra cómo los aportes del trabajo 
han caído dramáticamente en los últimos cinco años, arrastrando con ello el crecimiento del país. Aún más: pese a que en los últimos ranking de competitividad aparecemos liderando al resto de los países de América Latina, e incluso en un nivel bastante aceptable en comparación con países desarrollados y de rápido crecimiento como España, nuestro índice de productividad de la mano de obra -uno de los componentes de la competitividad que cuantifica el esfuerzo que debe hacer cada persona que trabaja para producir un dólar- nos ubica en los últimos lugares. En efecto, según el informe sobre competitividad, publicado el año 2001 por el International Institute for Management Development, que incluye a 49 países, Chile figura en el trigésimo sexto lugar, con un índice relativo al aporte de las personas al PIB por cada hora de trabajo, menor a 11,5 dólares la hora. En el caso de los países desarrollados este índice es tres veces superior. Esto muestra la urgente necesidad de adoptar medidas a fin de mejorar la capacidad de nuestros trabajadores para agregar valor en los procesos que desempeñan.

Por otra parte, en el mismo cuadro se observa que la PTF, medida como la parte residual del crecimiento del PIB, o el componente no explicado por los factores trabajo y capital, ha sido uno de los factores más importantes en el crecimiento del PIB en los últimos 25 años. Sin embargo, y tal como lo indica el último informe del Centro Nacional de la Productividad y la Calidad, actualmente dicha productividad no se está desarrollando a un ritmo que permita seguir creciendo a tasas sobre el $5 \%$ anual.

\section{Cuadro $\mathrm{N}^{\circ} 1$}

TASA DE

\begin{tabular}{lrrrr} 
CRECIMIENTO DEL & PIB & PTF & TRABAJO & CAPITAL \\
\hline $1976-1980$ & 6,8 & 3,2 & 2,4 & 1,2 \\
$1981-1985$ & $-0,1$ & $-2,3$ & 1,2 & 1 \\
$1986-1990$ & 6,5 & 2,2 & 2 & 2,2 \\
$1991-1995$ & 7,5 & 3,3 & 1,4 & 2,8 \\
$1996-2000$ & 4,6 & 1,6 & 0,5 & 2,5 \\
$1998-2001$ & 2,9 & 0,4 & 0,1 & 2,4 \\
\hline
\end{tabular}

Fuente: "Informe de productividad" en Revista del Centro Nacional de la Productividad y la Calidad, $N^{o}$ 9, Santiago, Chile, diciembre 2001, pp. 1-11 
Frente a estos dos aspectos, la educación superior tiene mucho que decir. En primer lugar, respecto de la productividad del trabajo, debido a que ella está íntimamente relacionada con la preparación de los profesionales y técnicos que serán los encargados de dirigir los procesos productivos de bienes y servicios, así como de entregar a la sociedad propuestas novedosas que aseguren su éxito. En segundo lugar, la educación superior incide en la PTF, puesto que ésta corresponde a la capacidad de incorporar las nuevas tecnologías en los procesos productivos, lo cual es factible de realizar por personas que hayan sido especialmente preparadas para este fin.

En Chile, la educación superior se organiza según la Ley Orgánica Constitucional de Enseñanza (LOCE) en: centros de formación técnica, que otorgan títulos técnicos de nivel superior; institutos profesionales, que otorgan títulos profesionales que no requieran licenciatura y universidades, que otorgan títulos profesionales y grados académicos. Esta organización pretende discriminar entre distintas instituciones que forman con diferentes orientaciones: unas, más orientadas al mundo laboral y sus necesidades, y otras a una actividad más académica -más forense-, que permite a cada una de ellas crecer en función de las necesidades reales del país.

Sin embargo, en la práctica, debido a un mal diseño en las políticas de financiamiento de la educación superior, se han establecido incentivos que atentan contra esa finalidad, de modo que las instituciones se han desarrollado en niveles que no corresponden a los requerimientos de las industrias. De acuerdo con las últimas cifras disponibles, publicadas por el Ministerio de Educación, en el año 2001 se matricularon 339.200 alumnos en universidades (227.284 en universidades del Consejo de Rectores y 111.916 en universidades privadas), en tanto que en los institutos profesionales lo hicieron 86.392 y en los centros de formación técnica 53.895.

Es evidente que la alta matrícula actual en las carreras universitarias, en comparación con las que registran los institutos profesionales y centros de formación técnica, no son proporcionales a los requerimientos de las empresas, que necesitan más personas preparadas para trabajos específicos, con una formación práctica y adecuadamente entrenados en las tecnologías actuales. Ello obliga a 
que profesionales universitarios deban desempeñarse en actividades y oficios para los que no tenían vocación ni fueron preparados, con el correspondiente derroche económico para el país y la entendible frustración para los jóvenes, que esperaban poder desempeñarse en otro tipo de labores.

Al efecto, conviene tener presente que esta situación es sustancialmente diferente en países desarrollados. Así por ejemplo en EE.UU., en los community colleges, instituciones que ofrecen carreras de dos años, semejantes de alguna manera a nuestros centros de formación técnica, estudian más de 10 millones de alumnos, cifra que, si se extrapolara para Chile, considerando a las poblaciones de ambos países, indicaría que aquí debería haber unos 500 mil cupos para técnicos de nivel superior, es decir, unas ocho veces los actuales, puesto que actualmente existen menos de 60 mil.

Con base en estos antecedentes es posible concluir que sería conveniente contar con los incentivos adecuados para que el crecimiento de la educación superior en Chile -la cual en los próximos 10 años debería contar con unas 300 mil plazas adicionales*, para alcanzar la cobertura de países con similares niveles de desarrollo- se produzca en las áreas técnicas y profesionales, más que en las carreras universitarias tradicionales. Sólo así sería posible asegurar un sistema educacional orientado a la formación de los cuadros técnicos y profesionales que el país requiere para asegurar el desarrollo.

* Véase bibliografía final. 


\section{ReFERENCIAS Bibliográficas y Notas}

Gallart, Maria Antonia y Jacinto, Claudia, "Competencias laborales: tema clave en la articulación educación-trabajo"; en Boletín de la Red Latinoamericana de Educación y Trabajo, CIID-CENEP, Año 6, $N^{o} 2$, Buenos Aires, Argentina, 1995.

Ibarrola, María de, "Educación básica y competencias laborales"; en el seminario: Lo público y lo privado en la formación para el trabajo; Fundaciones FES, Corona y Restrepo Barco, Consejo Gremial Nacional, Misión de Educación Técnica, Tecnológica y Formación Profesional, Bogotá, Colombia, 1998. (Véase texto completo en www.cinterfor.or.uy).

"Informe de Productividad"; en Revista del Centro Nacional de la Productividad y la Calidad, Nº 9, Santiago, Chile, diciembre 2001.

* Discurso del Presidente de la República, Don Ricardo Lagos Escobar, con motivo de la inauguración de la sede Alameda del Instituto Profesional DuocUC. 